\title{
Comparative Study on Early Warning Methods of Infectious Diseases
}

\author{
Song-nian $\mathrm{HU}^{1}$, Xiao $\mathrm{CHENG}^{1,2^{*}}$, Dan $\mathrm{CHEN}^{1,2}$ \\ ${ }^{1}$ School of Management, Hubei University of Chinese Medicine, Wuhan, Hubei, 430065, China \\ ${ }^{2}$ Key Projects Sciences Research Base of Humanities and Social in Hubei Province the Development Research Center of Traditional \\ Chinese Medicine, Wuhan, Hubei, 430065, China
}

\begin{abstract}
Major epidemics of infectious diseases will not only endanger people's lives and property, but also cause panic and social unrest. Therefore, it is particularly important to establish an infectious disease early warning system and take effective measures in time to prevent infectious disease outbreaks. The article summarizes the relevant definitions of infectious disease early warning system, domestic and foreign development status, infectious disease early warning models and methods, and aims to provide references for the establishment of infectious disease early warning systems.
\end{abstract}

\section{Introduction}

In recent years, there have been numerous public health emergencies around the world. Infectious diseases will bring greater harm due to their spreading speed. After the first outbreak of COVID-19 in Wuhan in 2019, it has spread to a global epidemic. According to statistics from the official website of China 's Health Commission, as of 24:00 on December 1, 31 provinces (autonomous regions, municipalities) and the Xinjiang Production and Construction Corps reported that a total of 81649 cured and discharged cases, a total of 4634 deaths, and a total of 86,551 confirmed cases have been reported,and a total of 881,135 close contacts have been tracked, and 6,753 close contacts are still under medical observation. Therefore, constructing an indicator system, collecting relevant data, and establishing predictive models for early detection of infectious disease outbreaks and timely warnings, so as to take countermeasures, are currently important means of infectious disease prevention and control. The author has formed the research foundation of this article by searching the "Infectious Disease Early Warning", "Infectious Disease Early Warning Model", and "Infectious Disease Early Warning Index" in databases such as CNKI, Wanfang, and Weipu.

\section{Early warning related concepts}

Early warning refers to the situation where the evidence of its causality and reaction relationship cannot be determined before the event occurs. Based on the collected information, comprehensive assessments are made on the location, scale, nature, influencing factors, radiation scope, degree of harm, and possible consequences of the predicted possible event. Afterwards, within a certain range, an appropriate method is adopted to issue warnings of incident threats in advance and take corresponding levels of actions to prevent the occurrence and development of incidents to the maximum extent ${ }^{[1]}$.

Infectious disease early warning refers to the issuing of warning signals before and in the early stage of an outbreak or epidemic of an infectious disease to remind the outbreak or epidemic of the possible risk of possible expansion ${ }^{[2]}$. The principle is based on the monitoring data, through the analysis and calculation of the early warning model, from the infectious disease monitoring data, discover and identify the abnormal situation of the infectious disease that exceeds the expected normal level. Internationally, there are different terms describing the early warning of infectious diseases, the most commonly used are "outbreak detection", "abnormal detection" and "early warning".

An effective infectious disease early warning system includes five basic elements: early warning target setting, early warning data source collection, early warning model detection and analysis, early warning information release and early warning signal response ${ }^{[3]}$.

Early warning indicators are indicators with potential early warning value, and the fluctuation range of the indicators is related to the epidemic or outbreak of the disease ${ }^{[1]}$. Once the fluctuation range of the indicator exceeds the prescribed warning line, a warning can be issued and the corresponding epidemiological investigation or intervention can be initiated. Therefore, early warning indicators need to have the characteristics of timeliness, accuracy, scientific and practicality.

\footnotetext{
${ }^{*}$ Corresponding author's e-mail: chengxiao@hbtcm.edu.cn
} 


\section{Development status of early warning system}

\subsection{Development status abroad}

The Center for Infectious Disease Control and Prevention of the United States took the lead in the surveillance of infectious diseases in the late 1940s, and the number of countries that began surveillance of infectious diseases under the promotion of the WHO gradually increased ${ }^{[4]}$. Especially after the "911" terrorist attack and the anthrax postal incident in the United States in 2001, more people realized the importance of timely monitoring and early warning of public health emergencies ${ }^{[5]}$. At present, many countries around the world have explored and established early warning systems for various infectious disease outbreaks, and they have gradually developed relatively mature. For example, RSVP ${ }^{[6]}$ mainly passively collects clinical medical workers' judgment information on patient symptoms, mainly collecting flu-like symptoms, fever with rash, fever with suspicious central nervous system inflammation, and severe diarrhea (Including water samples and bloody diarrhea), adult respiratory distress syndrome and acute hepatitis six categories of information. ESSENCE II ${ }^{[7]}$, actively collect some data generated by patients in the process of seeking medical treatment and some medical-related behavioral information of patients, including sensitive medical information, public information and data from other monitoring systems. RODS ${ }^{[8]}$, based on the network, mainly collects information on main complaints from hospitals, sales data of over-the-counter drugs, laboratory inspection information and poison control center telephone information, and displays real-time monitoring results to users in the form of charts.

The establishment of these early warning systems plays an important role in collecting data for the government and administrative departments to take timely measures against the epidemic, and also provides a reference for China to establish and improve an infectious disease early warning system.

\subsection{Domestic development status}

The early warning of infectious diseases in China started late, but it has developed rapidly after the SARS outbreak in 2003. The government attaches great importance to emergency preparedness and related legislation, and has issued "Regulations on the Management of Public Health Emergencies", "National Public Health Emergencies Emergency Plan", "Health Emergency Personnel Equipment Catalog (Draft)" and other documents. The staff and researchers of the CDC and medical colleges have done a lot of research on the early warning of infectious diseases from different diseases and different methods, mainly involving in SARS, hemorrhagic fever with renal syndrome, tuberculosis, hepatitis A, hepatitis B, malaria and other key infectious diseases.

In 2004, my country established the National Statutory Infectious Diseases Direct Reporting System (CIDARS), which uses modern communication methods to achieve networking with local medical institutions based on the information networking of disease prevention and control institutions in the country, province, city, and county. It can automatically warn and respond to system data sources, report and monitor diseases and events, and is relatively uniform, efficient, fast and accurate. At present, China has early warning and monitoring systems including influenza monitoring, AFP monitoring, unexplained pneumonia monitoring, and public health emergency reporting systems.

\section{Early warning models and methods}

The infectious disease model is to study the transmission speed, space range, transmission route, dynamic mechanism and other issues of infectious diseases to guide the effective prevention and control of infectious diseases. Through searching the literature, we found that there are many models and methods for early warning of infectious diseases. Early warning can be divided into qualitative early warning, quantitative early warning and comprehensive early warning.

Quantitative early warning is mathematical model prediction. It refers to the establishment of a suitable model to simulate the epidemic process of the disease in the population, reveal the relationship between various variables in the process of disease occurrence, and predict the future incidence level to explore the law of development ${ }^{[9]}$. The main early warning methods include time series analysis, gray model GM, wavelet model, and regression prediction model. Time series is the most commonly used method. See Table 1 for details.

Table1. Common Models or Methods for Quantitative Early Warning

\begin{tabular}{|c|c|c|}
\hline Name & Definition or principle & Features or applicability \\
\hline $\begin{array}{ll}\text { Time } & \text { series } \\
\text { analysis } & \\
\text { (ARIMA) } & {[10]}\end{array}$ & $\begin{array}{l}\text { The time sequence formed when the } \\
\text { forecast target changes with time is taken } \\
\text { as a random queue. There are mainly } \\
\text { moving average method, exponential } \\
\text { smoothing method (ES) and Box-Jenkins } \\
\text { model }\end{array}$ & $\begin{array}{l}\text { It is mainly used to analyze and solve the } \\
\text { stationarity, randomness and seasonality of } \\
\text { the time series. It can also predict the } \\
\text { development trend of the research object } \\
\text { based on the analysis of the time series. Data } \\
\text { preparation and operation are relatively } \\
\text { simple and easy to implement, and the results } \\
\text { are relatively accurate, and are usually used } \\
\text { to predict short-term fluctuations of } \\
\text { infectious diseases. The disadvantage is that } \\
\text { for rare and severe diseases, it cannot be well } \\
\text { simulated. }\end{array}$ \\
\hline
\end{tabular}




\begin{tabular}{cll}
\hline gray & model & After turning irregular raw data into more \\
(GM) & {$[11]$} & $\begin{array}{l}\text { regularly generated data, model equations } \\
\text { are established to predict future } \\
\text { development trends. }\end{array}$
\end{tabular}

Wavelet model ${ }^{[12]}$ One of the multi-factor models

Regression
prediction model
$[13]$

A method of predicting how a variable changes with other variables, in the absence of an outbreak, uses the distribution of residuals to determine the threshold. It is mainly divided into linear regression and nonlinear regression. The common ones are linear regression model, quadratic curve model, cubic curve model, and exponential curve model.
It has no strict requirements on the sample size and probability distribution, the model is simple, and the prediction effect is good.

Data and disease data that change in the form of an exponential function are more applicable.

It is suitable for short-term prediction of diseases with stable epidemic factors.

It is suitable for the analysis and application of discrete infectious disease time series data, especially seasonal infectious disease time series data.

It can be used for outbreak detection based on laboratory results and cases reported by the surveillance system, as well as for symptom monitoring.
Qualitative early warning refers to analyzing the development law and related factors of the disease, and judging the trend and intensity of the epidemic ${ }^{[14]}$. The main early warning methods include control chart method, ratio chart method, fuzzy mathematics theory, Markov chain prediction method, etc. See Table 2 for details.

Table2. Common Models or Methods for Qualitative Early Warning

\begin{tabular}{lll}
\hline Name & Definition or principle & Features or applicability \\
\hline Control chart ${ }^{[6]}$ & Using historical data and taking into account & It is suitable for all kinds of infectious \\
& the sensitivity, specificity and positive diseases, and has better effect on \\
& predictive value of indicators, by establishing infectious diseases with seasonal \\
& an upper or lower limit of indicator early epidemic or periodic epidemic law.
\end{tabular}

Markov chain prediction method ${ }^{[16]}$ Moving percentile
method (MPM)

Exponential weighted moving average

(EWMA) [15]
Ratio Chart $^{[15]}$ warning as a threshold for identifying abnormal signals, an early warning can be issued when the actual monitored data "out of control".

Calculate the fluctuation range of the average monthly incidence rate of a certain disease in 5 years, and draw a sense bar graph to predict the monthly incidence rate. In comparison, if the ratio exceeds this upper limit range, it means that there is a sign of epidemic.

Regarding time series as a random process, it is a way to predict the future through different initial states of things and apply probability theory to study the changing trend of random events.

Non-parametric test method. The median of historical monitoring data represents the average level of monitoring indicators, and the percentile of a certain confidence level is used as the control limit.

Sequence data processing method

It is suitable for infectious diseases whose incidence is normally distributed.

Both time and state are discrete

Early warning model based on time dimension

It is suitable for monitoring sequences with a relatively stable baseline, and is mostly used for short-term historical data. It can introduce weighting ideas based on the distance between historical data and current time, consider the correlation between past data and current data, and is highly sensitive to small changes, and can quickly identify outbreaks.

cumulative sum Continuously accumulate the difference control chart between the observed value and the expected
Real-time surveillance of outbreaks with small changes in the number of 


\begin{tabular}{|c|c|c|}
\hline$(\mathrm{CUSUM})^{[15]}$ & $\begin{array}{l}\text { value, gradually accumulate the fluctuations in } \\
\text { the data, and quickly and sensitively detect the } \\
\text { small abnormalities in the data. }\end{array}$ & $\begin{array}{l}\text { cases and surveillance of relatively } \\
\text { uncommon infectious diseases are } \\
\text { effective. Do not rely on long-term } \\
\text { historical baseline data. }\end{array}$ \\
\hline
\end{tabular}

The qualitative forecasting method is simple and easy to understand, mainly for forecasting popular trends, while the quantitative forecasting method is complicated and the forecasting accuracy is high. The more accurate the method, the higher the data requirements and the more complicated the calculation. The comprehensive early warning mainly refers to the application of two or more prediction models to predict a certain infectious disease ${ }^{[16]}$. In practical applications, more methods combine qualitative forecasting and quantitative forecasting, which overcomes the unity and generality of forecasting methods and improves the accuracy of forecasting results.

Transmission dynamics models can be divided into SI, SIR, SIRS, SEIR models, etc. according to the types of infectious diseases ${ }^{[13]}$. In general, the population within the epidemic range of infectious diseases is divided into several categories: the first is category S, Susceptible, which refers to those who are not infected but lack immunity and are susceptible to infection after contact with infected persons; the second is category E, Exposed, which refers to a person who has been in contact with an infected person but is temporarily unable to infect other people. It is applicable to infectious diseases with a long incubation period; The third is category I, Infectious, which refers to people who have contracted infectious diseases and can spread to members of category $\mathrm{S}$ and turn them into members of category E or I; the fourth category is category R, Recovered, which refers to a person who is quarantined or has immunity due to illness. If the immunization period is limited, members of the $\mathrm{R}$ class can revert to the $\mathrm{S}$ class.

In actual application, you can add or delete the corresponding parameters according to the specific conditions of the data, set the relevant situation yourself, and establish a suitable model for simulation and analysis. In addition, geographic information systems and remote sensing technologies, as emerging high-tech technologies, have been widely used in the prediction of infectious diseases and public health emergencies in recent years ${ }^{[17]}$.

\section{Discussion}

In recent years, China has actively explored the early warning of infectious diseases, including early warning indicators and systems, early warning models and methods. However, research started late, and there are still some shortcomings, mainly related theories, methods and technical systems still need to be continuously improved; there are too many model methods, and each model method has its applicable diseases and data types. No statistical technique can ideally adapt to every disease, so it needs to be considered comprehensively in practical applications. The second is that early warning of infectious diseases requires the fusion of various technologies, so further exploration is needed in this regard, such as computer technology, geographic information, remote sensing technology, modern biotechnology.

\section{References}

1. Yan Weirong. Research on the early warning index system of infectious diseases and three prediction models [D]. Huazhong University of Science and Technology, 2008.

2. Yang Weizhong, Lan Yajia, Li Zhongjie. Review and prospects of infectious disease early warning research [J]. Chinese Journal of Preventive Medicine, 2014, 48(4) 244-247.

3. Yang Weizhong. Early warning theory and practice of infectious diseases [M]. Beijing: People's Medical Publishing House, 2012: 2-3.

4. Zhang Liming, Zhang Xiaoxin. U.S. Public Health Rapid Response Mechanism and SARS Prevention and Early Warning System[J]. Chinese Hospital Management, 2003(07): 60-62.

5. Ren Jianping, Guo Qing, Gu Yaming. Talking about the construction of my country's public health information system from the health emergency mechanism in the United States $[\mathrm{J}]$. Health Soft Science, 2005(06)407-409.

6. Ma Fen, Wang Li, Li Hui. Research progress in early warning methods of infectious diseases[J]. Health Research, 2008(02)249-251.

7. Yu Erman. Preliminary discussion on the early warning indicator system of influenza outbreak[D]. Zhengzhou University, 2012.

8. Huang Zhao. Research on abnormal detection and prediction of the incidence of hand, foot and mouth disease in the Pearl River Delta [D]. Guangdong Pharmaceutical University, 2019.

9. Wang Binggang, Qu Bo, Guo Haiqiang, et al. Research on mathematical model of infectious disease prediction[J]. Chinese Health Statistics, 2007(05)536-540.

10. Liu Gang, Shan Fuxiang. ARIMA model and its application in predicting the incidence of measles[J]. Journal of Mathematical Medicine, 2011, 24(4): 379382.

11. Feng Dan, Luo Yanxia. Grey prediction model of epidemic characteristics of epidemic cerebrospinal meningitis. Journal of Mathematical Medicine, 2003, 16:97-99.

12. Wang Ping. Application of three prediction models in the prediction of the incidence of major infectious diseases[D]. Zhejiang University, 2010.

13. Yu Yanni, Nie Shaofa, Liao Qing, etc. Research progress in infectious disease prediction and model selection[J]. Public Health and Preventive Medicine, 
2018, 29(05)89-92.

14. Bradley C A, Rolka H, Walker D, et al. BioSense: Implementation of a national early eventdetection and situational awareness system[J]. Mmwr Morbidity \& Mortality Weekly Report,2005, 54(Suppl): 11-19.

15. Liu Wanyu. Research on the monitoring and early warning system of infectious disease symptoms in the army [D]. Chinese Academy of Military Medical Sciences, 2015.

16. Hu Shixiong, Xing Huixian, Deng Zhihong. The current situation of the prediction and early warning of infectious diseases in my country[J]. Chinese Journal of Preventive Medicine, 2007, 41(05) 407410.

17. Lu Feng Da, Yang Qifa, etc. Disease Surveillance Geographic Information System and its application in Yuhang District, Hangzhou [J]. Chinese Public Health, 2007, 23(9): 1137-1138. 